Please quote as: Barev, T. J.; Janson, A.; Leimeister, J. M. (2020): Designing Effective Privacy Nudges in Digital Environments: A Design Science Research Approach. International Conference on Design Science Research in Information Systems and Technology (DESRIST) (p/pp. 388-393) 


\title{
Designing Effective Privacy Nudges in Digital Environments: A Design Science Research Approach Research-in-Progress
}

\author{
Torben Jan Barev ${ }^{1(\bowtie)}$, Andreas Janson ${ }^{2}$, and Jan Marco Leimeister ${ }^{1,2}$ \\ ${ }^{1}$ University of Kassel, Kassel, Germany \\ \{torben.barev, leimeister\}@uni-kassel.de \\ ${ }^{2}$ University of St.Gallen, St.Gallen, Switzerland \\ \{andreas.janson, janmarco.leimeister\}@unisg.ch
}

\begin{abstract}
When using digital technologies, various data traces are left behind for collection, storage and analysis. Innovative solutions for information systems are needed that mitigate privacy risks and foster information privacy. One mechanism to achieve this is using privacy nudges. Nudges are a concept from behavioral economics to influence individual's decisions. However, many nudges show low or at least less effects than choice architects hope for and expect. Therefore, this design science research (DSR) project focusses on developing evidence-based design principles for privacy nudges to improve their effectiveness and pave the way for more privacy sensitive IT systems. In this context, we adopt a DSR approach from Vaishnavi \& Kuechler. From a theoretical perspective, we are contributing to the discussion of what drives privacy sensitive behavior. We extend generic nudge design models, making them applicable in the context of data disclosure. For practitioners, we provide guidance on how to design and implement effective privacy nudges in the user interface of digital work systems.
\end{abstract}

Keywords: Privacy nudging $\cdot$ Information privacy $\cdot$ Design science research

\section{Introduction}

Digital work environments are ubiquitous nowadays and the possibility to electronically acquire information about work activities as well as personal sensitive data has dramatically increased. Companies use more forms of digital work systems and implement advanced instruments such as big data analytics or artificial intelligence. With this, data can be collected, aggregated, and analyzed at a faster pace and in larger volume than ever before. This can lead to a systemic disadvantage, as an information asymmetry between the individual and the data processor exists [6]. The vulnerability to discrimination, commercial exploitation and unwanted monitoring is ubiquitous. Thus, the acceptance and adoption of modern IT systems is hindered.

The issue arising is that people value their privacy while they do not always protect it; this phenomenon is known as the Privacy Paradox [2]. As privacy is a critical antecedent 
for the acceptance of future work systems [5, 7] innovative solutions for information systems (IS) are needed that mitigate privacy risks and foster information privacy. A possible solution would embody privacy-by-design systems which are privacy enhancing technology components [10].

One mechanism to achieve this is the implementation of privacy nudges. Nudges are described as "any aspect of the choice architecture that alters people's behavior in a predictable way without forbidding any options, or significantly changing their economic incentives" [12]. Thus, privacy nudges should help users to make better privacy decisions [1]. However, some nudges emerge to have little or no impact on actual behavior [11]. Hence, in our design science research (DSR) project we are deriving evidence-based design principles that support decision architects to design effective privacy nudges. Our developed artifact should then answer the following research question: What are key design principles for privacy nudges leading users to a more privacy friendly behavior? To achieve our research goal, we selected an established and eminent DSPR approach that fits our purpose exactly. We follow the design science approach following Vaishnavi $\&$ Kuechler [14] (see Fig. 1).

\begin{tabular}{|l|l|l|l|l|}
\hline & $\begin{array}{c}\text { General Design Science } \\
\text { Cycle }\end{array}$ & Design Cycle One & Design Cycle Two \\
\hline
\end{tabular}

Fig. 1. Three consecutive design cycle and research activities.

In line with our research approach (Fig. 1), we have addressed the problem identification and motivation phase of the design science approach in this introduction. The remainder of the paper is organized to address the theoretical background of privacy nudging, the development of our design principles and evaluation. The paper closes by presenting a conclusion and contribution regarding the expected results [3, 4].

\section{Related Work}

\subsection{Privacy Nudging}

Particularly in the context of information-privacy-related decisions, human decisionmaking is often imperfect. Nudging is a promising approach, to enable individual users of digital systems to make decisions in line with their objectives pursued for their own data protection [5]. In digital environments, nudging typically uses design elements in the user interface to influence behavior [17]. Privacy nudges are a sub-form, describing a targeted influence on the decision-making process in order to lead people to privacy friendly decisions and guide individuals to informational self-determination $[1,9,17]$. 


\subsection{Related Methods for Designing Nudges}

To influence decisions and change behavior with nudges, various researchers proposed models of how to craft nudges $[5,8,17]$. Weinmann et al. [17] highlight for example how designers can create digital nudges and the authors developed a design cycle [17]. Another approach to provide an easier access to digital nudging is proposed by Meske and Potthoff [8] named the Digital Nudging Process Model (DINU Model). In this model the creation of digital nudging is divided into the three generic phases (1) Analyzing, (2) Designing, and (3) Evaluating including a feedback loop [11]. Linking to this, Mirsch et al. [5] proposed the Digital Nudge Design Method (DND-method) presenting a universal four-step approach of how to systematically design digital nudges [5]. These approaches tend to give mainly generic guidance of how to systematically craft digital nudges. Hence, as nudge effectiveness is highly context-dependent, these models can only serve as a scaffold. For the design and implementation of effective nudges they should be enhanced by specific characteristics of the decision environment and adapted to the privacy nudge context. On the same note, the presented models do not ensure that ethical and moral directives are thoroughly taken into account, making it prone for practitioners to design societally reprehensible nudges [18].

\section{Deriving Evidence-Based Design Principles}

\subsection{Research Objective and Artifact}

Our DSR project takes an interdisciplinary approach to develop an artifact representing a set of evidence-based design principles that support decision architects to design and implement effective privacy nudges. Privacy nudges are effective when ensuring individual's informational self-determination and lead users to a more privacy sensitive behavior. Our approach focuses on working out components of effective privacy nudges, which are assembled by the below presented design requirements. In the next step, to provide concrete guidance for nudge architects, we deduct specific design principles. Evaluating these principles in lab and real-world contexts, we generate fine granular evidence-based design principles for privacy nudges.

\subsection{Design Requirements}

To craft design requirements for designing effective privacy nudges, we conducted a systematic literature review and semi-structured explorative interviews with German industry representatives $(n=23)$. More than 50 design requirements have been collected, tested for redundancy and encapsulated to 9 tentative design requirements (Table 1). We did this in collaboration with an interdisciplinary expert group, ensuring that various perspectives are brought together. Information System (IS) knowledge, public law and IS law is brought in by two departments of a German Institute of IS Design. Ethical and philosophical knowledge is represented by an international center for ethics. Current industry knowledge for engineering future work systems is represented by two experts from a German institute for industrial engineering. After carving out the design requirements and consequential design principles, these components were condensed and simplified for easier understandability ensuring utility for choice architects. 
Table 1. Design requirements for privacy nudges in digital work systems.

\begin{tabular}{l|l}
\hline Design Issue & Derived requirement \\
\hline Usability & $\begin{array}{l}\text { DR1) Avoid slowing down work processes } \\
\text { DR2) Ensure quick and easy decision making }\end{array}$ \\
\hline Transparency & $\begin{array}{l}\text { DR3) Avoid manipulation and ensure ethical standards } \\
\text { DR4) Provide transparent information about the decision's consequences }\end{array}$ \\
\hline Interaction & $\begin{array}{l}\text { DR5) Avoid cognitive overload by difficult to process nudges } \\
\text { DR6) Avoid nudges that foster distraction and stress for users }\end{array}$ \\
\hline Adaptability & DR7) Consider target group's characteristics and needs \\
\hline Economy & $\begin{array}{l}\text { DR8) Do not endanger the company's business model } \\
\text { DR9) implementation of privacy nudges should be cost-effective }\end{array}$ \\
\hline
\end{tabular}

\subsection{Tentative Design Principles for Privacy Nudges}

From the developed design requirements, we are now able to craft design principles for the design of privacy nudges. We conducted a systematic literature review following a methodology proposed by vom Brocke et al., as well as Webster and Watson $[13,16]$ and identified 38 relevant paper. To extent and validate our results we conducted an expert workshop with the same consortium as described above. The preliminary results propose design requirements that effective privacy nudges in modern work system should meet. An extract of the design principles is listed below (Table 2):

Table 2. Tentative design principles for privacy nudges.

\begin{tabular}{l|l}
\hline Design requirement & Design principles \\
\hline Usability & DP1) Ensuring ergonomic and simplified design \\
& DP2) Smoothly integrate Nudges into work processes \\
& DP3) Focus on relevant information for privacy friendly decision \\
\hline Transparency & DP4) Give information of why and what the nudge is designed for \\
& DP5) Balance privacy and economic interest information \\
\hline Interaction & DP6) Use default and framing nudges preferentially \\
& DP7) Make privacy and privacy risks tangible \\
\hline Adaptability & DP8) Personalize nudges \\
\hline Economy & $\begin{array}{l}\text { DP9) Allow company's business model to function } \\
\text { DP10) Ensure design and implementation costs for feasibility }\end{array}$ \\
\hline
\end{tabular}

Using these design principles, an exemplary privacy nudge is presented below. Here, the nudge focuses on presenting relevant information for privacy friendly decision. The nudge is personalized, framed and uses a default. It can be smoothly integrated in the user interface of a digital work system (Fig. 2). 


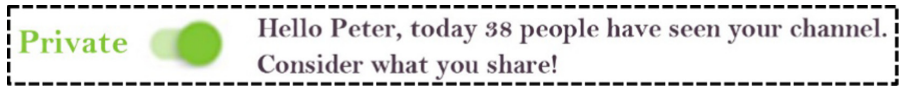

Fig. 2. Exemplary privacy nudge developed by deploying the design principles.

For further development, we break down the developed design principles into fine granular design theory. For illustration, DP8 can be further broken down in its components of e.g. personality traits, competencies or even emotions. These components will then be empirically evaluated resulting in evidence-based design principles.

\section{Evaluation and Future Outlook}

We will evaluate our artifact ex post in the fourth phase of each design cycle [15] following the Framework for Evaluation in Design Science (FEDS) proposed by Venable et al. [15]. The FEDS evaluation design process consists of four steps: (1) By taking a formative evaluation approach we use empirically based interpretations to rigorously demonstrate and improve utility, quality and efficacy of our design artifact. We test how well the artifact serves its main purpose of supporting choice architects to design and implement effective privacy nudges. (2) In a first step, we take an artificial evaluation approach by introducing and educating our design principles to students with a background in IS. Having our artifact at hand, the students are required to design privacy nudges. Afterwards, we assess the effectiveness of the crafted privacy nudges. We adopt a human risk and effectiveness evaluation strategy to rigorously determine whether the benefits of the artifact will remain in real-world digital work systems over the long run or not. (3) In order to determine the properties to evaluate we adapt levels of granularity proposed by Sun and Kantor [19]. We evaluate whether the task of crafting privacy nudges was completed and whether the designed nudges have an impact on user's behavior of disclosing personal data. (4) In total, two evaluation episodes are planned with the student group. The evaluation is complemented by a naturalistic field experiment and, in the end, a summative evaluation of the artifact.

\section{Contribution and Conclusion}

With the artifact representing evidence-based design principles for privacy nudges, our contribution is twofold. From a theoretical perspective, our nascent design theory contributes to the discussion of what drives privacy sensitive behavior. We extend existing generic models, making them applicable in the context of data disclosure. For practitioners, we offer evaluated and specific design-directives-crafting privacy nudges. Importantly, our artifact is abstracted ensuring the adoption of our design principles to all types of privacy nudges. Even though some design principles seem promising to adapt in different applications, we can only offer evidence-based design knowledge in the environment of privacy decision making and data disclosure behavior. However, transferring and testing them in a different application environment may represent a fruitful endeavor in the future. 
Acknowledgement. The research presented in this paper was funded by the German Federal Ministry of Education and Research in the context of the project Nudger (www.nudger.de), grant no. $16 \mathrm{KIS} 0890 \mathrm{~K}$.

\section{References}

1. Acquisti, A., Sleeper, M., Wang, Y., Wilson, S., Adjerid, I., Balebako, R., et al.: Nudges for privacy and security. ACM Comput. Surv. 50(3), 1-41 (2017)

2. Barth, S., de Jong, M.D.T.: The privacy paradox: Investigating discrepancies between expressed privacy concerns and actual online behavior - a systematic literature review. Telematics Inform. 34(7), 1038-1058 (2017)

3. Erevelles, S., Fukawa, N., Swayne, L.: Big data consumer analytics and the transformation of marketing. J. Bus. Res. 69(2), 897-904 (2016)

4. Cazier, J., Wilson, E., Medlin, B.: The role of privacy risk in IT acceptance. Int. J. Inform. Secur. Priv. 1(2), 61-73 (2007)

5. Mirsch, T., Lehrer, C., Jung, R: Making digital nudging applicable: the digital nudge design method. In: International Conference on Information Systems, pp. 1-16 (2018)

6. Schwartz, P.M.: Property, privacy, and personal data. Harv. L. Rev. 117(7) 2056 (2003)

7. Paul, P., Integrating trust in electronic commerce with the technology acceptance model: model development and validation. In: AMCIS 2001 Proceedings. vol. 159 (2001)

8. Potthoff, T.; Meske, C.: The DINU-model - a process model for the design of nudges. In: 23rd European Conference on Information Systems (ECIS), pp. 2587-2597. Portugal (2017)

9. Schöbel, S.; Barev, T.J.; Janson, A.; Hupfeld, F., Leimeister, J.M.: Understanding user preferences of digital privacy nudges - a best-worst scaling Approach. In: Hawaii International Conference on System Sciences (HICSS) (2020)

10. Spiekermann, S.: Ethical IT Innovation: A Value-Based System Design Approach, 1st edn. CRC Press, USA, Florida (2015)

11. Sunstein, C.: Nudges that fail. Behavioural. Public Policy 1(1), 4-25 (2017)

12. Thaler, R.H., Sunstein, C.R.: Nudge. Int'l. Penguin Books, London (2009)

13. vom Brocke, J., Simons, A., Riemer, K., Niehaves, B., Plattfaut, R., Cleven, A.: Standing on the Shoulders of Giants: Challenges and Recommendations of Literature Search in Information Systems Research. In: CAIS 37 (2015)

14. Vaishnavi V. and Kuechler W.: Design science research in information systems. AISNet. In: Association for Information Systems. USA (2004)

15. Venable J.R., Pries-Heje R., Baskerville R.: FEDS: a framework for evaluation in design science research. IS Technol. 25(1), 77-89 (2016). Springer, Berlin

16. Webster, J., Watson, R.T.: Analyzing the past to prepare for the future: writing a literature review. MIS Q. 26(2), 13-23 (2002)

17. Weinmann, M., Schneider, C., vom Brocke, J.: Digital nudging: guiding online user choices through interface design. Commun. ACM 61(7), (2018)

18. Weinmann, M., Schneider, C., vom Brocke, J.: Digital nudging. Bus. Inform. Syst. Eng. 58(6), 433-436 (2016). https://doi.org/10.1007/s12599-016-0453-1

19. Sun, Y., Kantor, P.B.: Cross-Evaluation: a new model for information system evaluation. J. Am. Soc. Inf. Sci. Technol. 57(5), 614-628 (2006) 